\title{
THE PLACE OF VARIATION IN TEACHING OF FRENCH AS SECOND LANGUAGE IN THE UNIVERSITY OF BUEA IN CAMEROON
}

\author{
[LA PLACE DE LA VARIATION DANS L'ENSEIGNEMENT DU FLE \\ A L'UNIVERSITE DE BUEA AU CAMEROUN]
}

\author{
C. M. Gisele Piebop
}

doi: 10.18355/PG.2016.5.2.297-310

\begin{abstract}
Although an Anglo-Saxon university, the University of Buea is set apart by the practice and teaching of French in order to match with the individual and official bilingualism promoted by Cameroon. However, the French which is provided to non-native French speaking students in particular does not reflect endogenous standards. Fact that often confuses and discourages learners in the learning process. In other words, this study seeks to show that the extremely standardize variety prescribed for the teaching of French as foreign language seriously undermines the quality and effectiveness of teaching in a highly multi-lingual and multi-cultural country like Cameroon. This is why it is required to re-orientate the current syllabus and teaching methods on variation, in order to efficiently achieve the objectives sought by this course.
\end{abstract}

Key words

French as foreign language, variation, standard, cultures, practices

\section{Résumé}

Bien qu'étant une université de tradition anglo- saxonne, l'université de Buéa ne déroge pas pour autant à la pratique et l'enseignement du français en son sein; bilinguisme officiel et individuel du Cameroun obligent. Cependant, le français qui y est mis à la disposition des étudiants non natifs francophones en principe est loin de tenir compte des normes endogènes et de la variation en général. Fait qui très souvent dépayse les apprenants et les décourage dans l'apprentissage. En d'autres mots, cette étude cherche à montrer que le français hyper normé à la Vaugelas prescrit pour l'enseignement du français langue étrangère entame sérieusement la qualité et l'efficacité des enseignements dans un contexte hautement pluri et multi lingue/culturel comme celui du Cameroun. Raison pour laquelle l'urgence d'un réajustement des contenus où la variation aura une place de choix s'impose, en vue d'atteindre effectivement les objectifs convoités par ce cours.

Mots clés

Français Langue Etrangère, Variation, norme, cultures, usages.

\section{Introduction}

L'Etat camerounais définit clairement le type de Camerounais qu'il voudrait modeler à travers son système éducatif dans l'article 5 des dispositions 
générales de la loi n 98/004 du 14 avril 1998 d'Orientation de l'Education au Cameroun. Pour lui, l'éducation a pour objectif : «1) la formation des citoyens enracinés dans leur culture, mais ouverts au monde et respectueux de l'intérêt et du bien commun...» Et pour garantir les mesures d'accompagnement de cette disposition, l'article 11 portant sur l'élaboration et la mise en oeuvre de la politique et du financement de l'éducation précise : «[...] à cette fin, il (l'Etat) veille à l'adaptation permanente du système éducatif aux réalités économiques et socioculturelles [du pays], ainsi qu'à l'environnement international, particulièrement en ce qui concerne la promotion des enseignements scientifiques et technologiques, du bilinguisme et l'enseignement des langues nationales ». Il en ressort que les enseignements au Cameroun doivent sans cesse être adaptés aux contextes économiques et socio-culturels, surtout lorsqu'ils sont en relation avec la promotion du bilinguisme ou du multilinguisme au Cameroun, un des pans majeurs de la pressente étude. Or, il parait aisé de notifier que les manuels actuels d'enseignement du FLE à l'université de Buéa, à savoir Le Boulevard 1 pour le premier semestre et Le Boulevard 2 pour le second ne contiennent que des contenues exogènes, extravertis, qu'il s'agisse des concepts linguistiques ou sémioculturels. Tous les écrits, références et autres illustrations réfèrent au contexte européen et français précisément. Pourtant, ces cours sont destinés aux apprenants camerounais anglophones d'abord, puis francophones et étrangers au Cameroun. Il reste indéniable que le "Syllabus Review (1997)" de cette université a pour objectif de rendre les étudiants capables de communiquer à l'oral comme à l'écrit dans un environnement d'expression française. Pourtant, on en est à se demander quel est l'environnement direct dans lequel les étudiants sont sensés mettre en pratique toutes ces compétences liées à la France. Paradoxe des paradoxes, c'est le Cameroun qui est un pays différent de la France et qui de ce fait possède des réalités toutes aussi différentes des siennes. Se peaufine alors à l'horizon la nécessité d'accorder une place précieuse à la variation dans les enseignements, afin de garantir leur succès. Linguistiquement parlant, la variation renvoie à un phénomène sociolinguistique qui conçoit la langue comme un système complexe et non homogène, qui n'est jamais identique à deux époques ou lieux et qui est susceptible de changer selon des paramètres tels l'âge, le sexe, la catégorie socio-professionnelle... (Labov, 1976:103). Et justement, par le biais du variationnisme sociolinguistique qui permet d'étudier, et ce dans la pure tradition sociolinguistique, l'influence de variables extralinguistiques sur les pratiques des locuteurs, cette investigation démontrera l'apport de la prise en compte des usages dans l'enseignement du FLE à l'université de Buéa.

\section{La position de la France sur la variation dans l'enseignement du FLE}

Il y a belle lurette que les manuels d'enseignement de l'anglais langue étrangère ont cédé une place substantielle aux diverses variétés d'anglais tant en Grande Bretagne que partout ailleurs en Europe et sur les autres continents. Ce fait pourrait se justifier par l'attitude libérale de la Grande Bretagne à l'égard des différentes formes d'anglais, et qui du reste n'a jamais essayé de 
leur imposer une norme internationale. En effet, malgré l'existence de la prestigieuse "Received Pronunciation", les variantes régionales et dialectales d'anglais ne sont nullement dévalorisées. Contrairement à la Grande Bretagne, la France est reconnue pour son intolérance vis-à-vis des variétés dialectales et régionales du français. Il n'y a qu'à évoquer l'intitulé d'une enquête conduite par Grégoire entre 1790 et 1792, à savoir: "Sur la nécessité et les moyens d'anéantir les patois et d'universaliser la langue française », pour s'en faire une idée nette. Cette attitude est confortée par le désir de codification de la langue française pour en faire une norme internationale qui a toujours habité la France, et qui a d'ailleurs amené $F$. Brunot (1966: III, cité dans Lodge, 1993: 157) à déclarer que « le règne de la grammaire [...] a été plus tyrannique et plus long en France qu'en aucun pays ».

De la sorte, ce désir constant de faire respecter la norme du français va se transposer dans l'enseignement du Français Langue Etrangère, avec pour ultime objectif depuis les années 70 de faire acquérir des compétences communicatives, mais surtout linguistiques aux apprenants. Autrement dit, l'enseignement du FLE a pour dessein d'amener les apprenants à s'exprimer dans un français correct. Les adeptes de ce purisme font du français central une langue passe-partout dans le monde francophone, et se justifient en posant qu'un étranger qui s'exprime à travers le français standard est toujours compris et ses écarts sont mieux tolérés, au détriment de celui qui utilise une variante régionale ou stylistique plus ou moins maitrisée. Il s'en dégage une règle d'or d'après laquelle : il est préférable de parler trop bien que d'utiliser des variantes familières à mauvais escient. Ce choix peut s'avérer efficace dans un contexte où les réalités sont presque identiques, mais pose quelque peu des problèmes lorsqu'il est transposé ailleurs, particulièrement au Cameroun et dans une zone anglophone où d'autres realia ont cours et où les motivations et les conditions de déroulement des cours sont différentes. Encore que même en France, les spécialistes s'accordent de plus en plus sur la nécessité d'intégrer la variation dans l'enseignement du français, afin de le rentabiliser. Eloy (2003: 13) expose clairement cette position lorsqu'il déclare qu' "il faut renoncer à la fallacieuse sécurité intellectuelle de ceux qui ne veulent pas savoir la complexité de la langue. Il nous faut comprendre que la langue n'est pas seulement un système linguistique, mais sociolinguistique. Il nous faut accepter que la grammaire normative ne suffit pas ».

\section{Situation de l'existant sur l'enseignement du FLE à l'université de Buea}

Le français et l'anglais constituent des matières obligatoires pour tous les étudiants des universités du Cameroun, quel que soit la spécialité ou la filière. Ces langues sont dispensées dans le cadre de la formation bilingue où le français est dispensé aux Anglophones natifs et l'anglais aux Francophones. Mais particulièrement à l'université de Buéa, ces deux langues coulées dans les programmes de FLE décliné en français fonctionnel et d'anglais langue étrangère (use of English) sont imposées sans exception à tous les étudiants de première année. 
En rapport avec les manuels utilisés pour les cours de FLE à l'université de Buéa, on l'a dit, ils s'intitulent Le boulevard 1 réservé au cours de functionnal french 1 (FRE 101) au premier semestre et visant le niveau A1 du CECR et Le Boulevard 2 pour le cours de functionnal french 2 (FRE 102) au second semestre et prétend atteindre le niveau A2 du CECR. Ces manuels sont conçus pour autonomiser l'enseignement et l'apprentissage et son axés autour de textes régulièrement illustrés mettant en exergue des contenus communicatifs, d'une partie grammaire inhérente à l'objectif de communication et d'une autre partie qui vise l'acquisition des savoir-faire et des compétences.

Pour ce qui est du français fonctionnel ou FLE qui y a cours, il est initialement et prioritairement destiné aux étudiants anglophones qui pour la plupart se répertorient au niveau de débutants en français pour plusieurs raisons (pénurie d'enseignants de français au niveau antérieur, séchage de cours de français, antipathie pour le français...). A côté d'eux, on retrouve d'autres Anglophones ayant déjà été en contact en français et dont le niveau est acceptable, du moins à l'oral; soit parce qu'ils fréquentent ou viennent des zones francophones du pays, soit parce qu'ayant suivis avec assiduité leurs cours de français au primaire et au collège et de la sorte ayant optés pour le français au second cycle. Par ailleurs, la population estudiantine de l'université de Buéa est également constituée de Francophones qui désirent y compléter leurs études, de même que des étudiants étrangers (Ghanéens, des Nigérians, des Equato-guinéens...). C'est ainsi que l'on se retrouve avec des 300 locuteurs à des degrés L1, L2, L3, ... Ln dans le même cours de français. Ces multiples et différents profiles rien qu'à eux seuls font déjà naître en toile de fond la problématique de la variation. On peut mentionner à cet effet, les étudiants venant de la zone francophone, étant de ce fait en contact avec les autres variétés de français au Cameroun. Pour cette raison, ils créent constamment la polémique pendant les leçons. Dans l'unité ouvroir du manuel Le Boulevard I conçu pour le premier semestre portant sur la prise de contact par exemple, il est question de saluer et de demander des nouvelles formellement par bonjour monsieur/madame/mademoiselle ; comment allezvous ou encore informellement par bonjour + nom ou plus petit nom, salut... comment ça va ?/ comment vas-tu ? Pourtant certains étudiants s'offusquent de ce que des possibilités telles que bonjour mon frère/ma sœur ou bonjour papa/maman à des inconnus âgés, C'est comment/on dit quoi ?ça dose ? Pour dire comment ça va ? n'existent pas dans le manuel et ne soient de se fait pas acceptés. De même, ils font les mêmes revendications pour les expressions On est ensemble, on se voit, on se prend qui devraient figurées à côté de au revoir, bonne journée ou à la prochaine dans le manuel.

En réalité, ces expressions bénéficient en zone francophone du pays non seulement d'une grande fréquence, mais aussi d'une durée prolongée dans le temps, deux critères qui font d'elles des normes endogènes, ceci du fait de leur encrage dans le contexte socioculturel africain et camerounais précisément. En fait l'amour et la solidarité des Africains sont un secret de polichinelle qui font de tout le monde le parent de tout le monde. Voilà pourquoi ils préfèrent ces termes plus affectueux frère/sœur maman, papa à 
monsieur/madame/mademoiselle qui créent tout de suite une distance entre les interlocuteurs. Pareillement, les Africains sont toujours là l'un pour l'autre et quand bien même l'un meurt, selon les croyances animistes du terroir, il continue de veiller depuis le monde des morts sur les vivants, ce qui explique la célèbre boutade « les morts ne sont pas morts » de Birago Diop. Voilà qui justifie pourquoi les Camerounais disent général par transposition de leurs langues maternelles : On est ensemble, on se voit, ... car dire au revoir implique déjà l'idée de séparation, d'isolement, même à court terme.

Pris sous un angle plus pragmatique, l'expression bonjour madame/monsieur d'un acheteur à un vendeur ou une vendeuse et vice versa dans un marché par exemple est maladroit et prédispose une communication à un échec pour celui qui désire faire de bonnes affaires. Car en général, l'expression bounjour ass/asso, terme économique pour désigner l'associé et signifiant contextuellement fidèle des fidèles, établit sur le champ une proxémique favorisant la connivence et surtout « une véritable relation commerciale de convivialité qui préserve toute son humanité et refuse le privilégier le côté marchand. » (Modo Asse, 2010 : 149). Et plus l'on s'exprime en français normé, plus les prix des articles souhaités sont multipliés. Le marché est considéré comme un lieu de la débrouillardise où la plupart des vendeurs sont en général peu instruits et de ce fait font des usages, des jargons leur apanage. Alors parler le français standard devient un signe d'instruction, donc de richesse qui frustre les vendeurs; raison pour laquelle ils cherchent généralement à se faire du beurre sur la tête de ce genre de clients non avisé.

On pourrait aussi mentionner parti tant d'autres exemples, l'unité1 du Boulevard 2 qui parle des activités quotidiennes l'extrait « Un jour en hiver» qui développe le champ lexical de l'hiver à travers la journée de M. Lefort et aboutit à un contenu conceptuel pourtant sur les quatre saisons européennes que sont l'été, l'hiver, le printemps et l'automne, sans aucune mention pour les saisons du Cameroun ou d'ailleurs. A ce niveau, on pourrait se poser la question de savoir si les Anglophones qui sont les apprenants prioritaires de ce cours pourrait bien comprendre les saisons européennes sans savoir comment s'appellent et fonctionnent les saisons du pays dans lequel ils subissent l'apprentissage et sont de fait appelés à interagir en premier.

L'examen des manuels ne laisse pas entrevoir de contextualisation ou de variation dans la mise en forme des différents types de savoirs, des savoirêtre, des savoir-faire... Tout ou presque, en dehors de quelques noms de pays qu'impose la leçon portant sur les nationalités, y est concentré sur le seul contexte français, à la limite européen: les noms (Lefort, Ferro, Doré, Leblanc, Lévêque, Namur, Lausanne, Paris, Louvre, Madrid, Marseille...) en passant par les styles vestimentaires «Les vêtements, la mode » (pp. 19-21), les descriptions «Pour aller au Louvre?»(pp. 36-37), les récits «faits divers » (p. 37), les illustrations...

Il demeure vrai que l'homme est un globe-trotter et que le français central permet aux apprenants de FLE d'interagir avec tous les autres Francophones du monde, mais le fait est qu'en attendant de rencontrer ces autres Francophones, les étudiants de l'université de Buéa doivent s'exercer avec d'autres Camerounais qui eux ont taillés le français à leur mesure afin de s'y sentir à l'aise et risquent de les discriminer ou les exclure de leur 
communication. Jetés de la sorte dans cet environnement camerounais, ils sont plutôt dépaysés, du fait de l'inadéquation des notions apprises avec le vécu quotidien.

En fait, pour les apprenants anglophones et étrangers surtout, la transposition exacte des connaissances basées sur l'Occident reçues en classe s'averra impossible, étant entendu que la camerounisation du français est une mission que s'assigne le commun des Camerounais francophones, question d'enrichir le français et de traduire avec exactitude leurs réalités culturelles, quotidiennes et leur identité. Ce qui revient à dire que la communication ne sera pas toujours réussie comme elle l'aurait été si les approches avaient été ne serait-ce que contrastives ou alors variantes en classe. Cela est d'autant plus vrai que les locuteurs ne seront pas toujours aptes à reconnaître ce français des étudiants comme correct, à moins d'être des instruits. Et de fait, il deviendra difficile de décoder et d'encoder des messages de façon fidèle. $\mathrm{Vu}$ sous cet angle, la communication demeurera superficielle, car lorsqu'il faudra par exemple désigner les réalités vestimentaires qu'ils ont pourtant étudiées en classe, on décèlera dans leurs discours verbaux, non verbaux et para verbaux des signes d'insécurité linguistique, car ils n'auront pas les compétences suffisantes pour trouver les termes exacts (kaba, grelots, gandoura, mbila...) et pour cela, ils pourront se taire, bégayer, utiliser des termes approximatifs... Autrement dit, la non prise en compte de la variation ici entraîne à la fois l'insécurité linguistique et culturelle des apprenants, puisqu'ils se voient en classe interdits d'utiliser des tournures et des termes 302 pourtant acceptés dans les discours courants des Francophones du pays.

En rapport avec le temps imparti aux cours de FRE101 et FRE 102 à l'université de Buéa, il a été muté de 2 heures par semaine x 10 à 2 heures par semaine x 13 pour chaque semestre depuis le début de l'année universitaire 2015-2016. Soit 26 heures x 2 donc 52 heures, temps qui reste tout de même très court pour atteindre le seuil A2 de la formation, car au moins 80 heures sont recommandées par les méthodes de FLE prévus à cet effet. A cela, se superposent les effectifs exorbitants (75 à 80 étudiants par groupe/classe) qui transforment le travail de l'enseignant en un véritable chemin de croix, car il lui devient quasi-impossible de moduler et de contrôler la classe, sans compter que les bancs ou sièges sont inamovibles. Tous ces aléas ne sont bien évidemment pas de nature faciliter la pratique de la pédagogie de la variation aussi bien linguistique que culturelle, qui pour ce qui est de la dernière nécessite suffisamment de temps pour son évaluation. On le constate, plusieurs facteurs tels les niveaux épars et hétérogènes des étudiants, les manuels extravertis, les effectifs excessifs, l'insuffisance de temps... favorisent la non prise en compte de la variation dans l'enseignement du français langue étrangère à l'université de Buéa ; ce qui ne va pas sans engendrer des conséquences.

\section{Incidences de l'absence de variation}

L'usage exclusif du français standard dans l'enseignement du FLE dans une université camerounaise de tradition anglophone comme Buéa, implique d'autres facteurs qui mis ensemble contribuent à y impacter négativement 
l'apprentissage du français. Cet aspect en particulier constitue un réel obstacle à l'atteinte de l'objectif fonctionnel de ce cours qui, honnêtement parlant, ne l'a jamais été. Et de fil à aiguille, il en découle un nombre considérable de conséquences fâcheuses.

\section{Le manque d'engouement des apprenants}

La centration du cours de FLE sur le français central et un environnement socio-culturel strictement occidental a ceci de négatif qu'il démotive les apprenants non seulement anglophones, mais également francophones, voire étrangers. Certains Camerounais francophones désertent les cours du fait de la monotonie et parce qu'ils trouvent le niveau d'apprentissage (A1 et A2) trop bas pour les habitués du français qu'ils sont ; entraînant ainsi avec eux leurs amis de niveau basique qui comptent sur eux lors des tests. D'autres, Anglophones pour la plupart cette fois, le font à cause du caractère selon eux hyper normé et de ce fait difficile et anxiogène du français par rapport à l'anglais, qui ne leur paraît pas identique à celui que parlent leurs homologues francophones en leur compagnie. Par conséquent, il leur est difficile de s'exercer avec eux. Qui pis est, ils se sentent frustrés par des étudiants déjà calés en français et qui ne leur laissent pas le temps de réfléchir ou se moquent souvent d'eux. En plus, les nombreuses récriminations des Francophones pendant le cours sur les différences d'avec les situations réelles en contexte francophone et la non figuration dans les manuels des usages camerounais du français contribue également à démotiver un grand nombre d'étudiants à la participation aux cours. C'est pourquoi très souvent, ces étudiants s'absentent des cours malgré le bonus de 5 points de participation au cours qui leur sont officiellement attribué, et n'apparaissent que lorsque les évaluations sont annoncées par les enseignants. Face à cette situation, l'intervention de la pédagogie de la variation, appliquée même à un niveau sommaire en classe, pourrait leur éviter la monotonie et les amener à retrouver le goût de prendre part aux cours de français.

\section{La défaillance de compétences pragmatiques}

Il est clair et tous les manuels de méthode le signalent d'ailleurs, l'enseignement d'une langue étrangère ne peut être réussie sans de réels approfondissements sur l'histoire et la culture de cette langue. Ce qui pose problème à l'université de Buéa est moins le fait que la culture française soit toujours en avant, que l'implication de la socioculture ou du contexte des pays des apprenants de cette langue, c'est-à-dire ne serait-ce que le Cameroun. En d'autres termes, il s'agit de la pédagogie de l'interculturalité que l'on peut appréhender comme l'ensemble des processus psychiques, relationnels, groupaux, institutionnel... générés par les interactions de cultures, dans un rapport d'échange réciproques et dans une perspective de sauvegarde d'une relative identité culturelle des partenaires en relation. Ce qui fait cruellement défaut dans l'enseignement du FLE à l'université de Buéa et à ce titre, les objectifs ne sauraient être atteints. Les réalités socioculturelles ne sont pas par exemples comparées avec celles du Cameroun et d'autres pays... L'étudiant débutant ne saura donc pas, s'il lui arrivait d'interagir avec un autre locuteur, les équivalents camerounais des 
saisons européennes illustrées dans le manuel et qu'il a étudiés en classe. Pareillement, il pourrait éprouver des difficultés à distinguer le décompte manuel de l'Occident qui se fait en levant progressivement les doigts du pouce à l'auriculaire, de celui camerounais qui se fait plutôt en pliant les doigts initialement levés de l'auriculaire vers pouce, ou encore de celui chinois qui es différent des deux autres, etc. Cette défaillance des compétences pragmatiques orchestrée par l'absence de variation, voire de partenariat dans l'enseignement à Buéa et en Afrique en général ne date pas d'aujourd'hui, car Ngalasso (1990: 24) l'avait déjà diagnostiqué, raison laquelle il avait prévenu que «tant que les langues [et cultures] africaines seront bannies de l'espace de vie ordinaire, [...] il y aura toujours le problème de l'apprentissage et la maîtrise du français comme matière dispensée dans cette langue».

\section{La subsistance des préjugés}

En tant que croyances ou opinions préconçues, les préjugés relèvent des représentations, c'est-à-dire "l'ensemble des images, des positions idéologiques, des croyances qu'ont les locuteurs au sujet des langues en présence et des pratiques linguistiques, les leurs et celles des autres. » Calvet (2002 : 121). Et tant que le contact des hommes, des langues et des cultures existera, les hommes auront des manières particulières de se voir et de regarder les autres. L'introduction de langues étrangères et particulièrement la langue française au Cameroun n'est pas vue d'un bon œil par certains 304 Camerounais qui éprouvent du mal à passer l'éponge sur l'incident historique de la colonisation. Ils considèrent le français normé comme un instrument d'asservissement qui ne tient pas compte de leurs réalités. Ils doutent sur les bonnes dispositions d'esprit de la France et de l'Etat qu'ils considèrent comme l'agent de cette France et qui pour eux a toujours brillé par son hypocrisie. Raison pour laquelle ils demeurent sceptiques et nourrissent du désintérêt et de la haine quant à l'apprentissage du français (Piebop, 2015).

Qui plus est, l'absence de la variation dans les cours de FRE 101 et FRE 102 n'est pas de nature à rassurer les natifs anglophones qui restent continuellement fixés sur l'idée d'un complot fomenté contre eux du fait de leur statut minoritaire, avec deux régions sur les dix que compte le pays. Ils sont en permanence sur le qui vive et crient inlassablement à la marginalisation. Ils voient en cette initiative d'imposition du français, normé de surcroît, à tous les étudiants d'une université pourtant de tradition anglophone, un prétexte pour confirmer la rumeur d'annexion des régions anglophones à celles francophones contiguës du littoral et de l'ouest. Pour calmer les esprits, la résolution de ce problème avait nécessité la tenue d'un « grand débat » qui avait abouti en 2008 à la transformation des 10 provinces d'antan en 10 régions. Alors il va de soi que des manuels autant extravertis ne font que frustrer davantage la communauté native anglophone du pays et accroître davantage sa suspicion à l'égard de l'Etat.

\section{Les échecs scolaires et les tricheries}


L'option de la focalisation du cours de FLE sur le français de référence à Buéa produit de nombreux effets négatifs parmi lesquels on retrouve en bonne place la démotivation des étudiants qui se sentent aliénés. Et du coup, les conséquences sont visibles sur leurs rendements scolaires. Il n'est pas sans objet rappeler que de nature, les populations anglophones, principales cibles du cours, éprouvent de vives réticences, si ce n'est de l'antipathie quant à l'apprentissage du français. (Piebop, 2015) et éprouvent un certain naturel à légitimer cet état de choses à la moindre difficulté rencontrée en français, et ce à travers des expressions telles "We are not Francophones !», "You should understand!!!». Attitude réciproque d'ailleurs pour les étudiants francophones qui aiment à clamer que «C'est Dieu qui donne l'anglais ». Par conséquent, les niveaux dans ces deux langues sont bas aux examens de l'enseignement secondaire du sous système anglophone surtout, c'est-à-dire au GCE/OL (General Certificate of Education Ordinary Level). English et French figurent toujours parmi les matières qui enregistrent les plus grands tôt d'échec (Piebop, 2015). A l'Université de Buéa par exemple, les échecs au functional french sont parfois à la lisière du catastrophique. Pour un cours prévu en principe uniquement pour les premières années, le nombre de participants a oscillé de 2500 étudiants répartis dans 64 groupes/classes en 2008 à près de 5000 au cours de l'année universitaire 2012-2013 pour le même nombre de groupes, car en plus de nouveaux étudiants normalement inscrits en première année, nombreux se réinscrivent pour la nième fois parce que n'ayant toujours pas pu valider le cours.

A la vérité, valider ce cours devient pour les étudiants un moyen d'obtenir leurs diplômes et non d'approfondir leurs connaissances en français, surtout lorsqu'un facteur motivant comme la variation n'intervient pas pour les pousser à s'approprier cette langue et en tirer tous les atouts connexes dont elle regorge. Pour parvenir à leurs fins, ces étudiants se rendent fautifs de toutes sortes de pratiques ignobles telles que les tricheries en cascade et les mercenariats. Ces techniques sont surtout l'apanage des étudiants finissants qui ont besoin de valider cette unité de valeur coût que vaille pour achever leur cursus. Ils s'arrangent et parviennent à tricher (parfois en masse), ce malgré la surveillance pointilleuse, sur les copies de leurs camarades qu'ils ont au préalable identifiés comme érudits en français. Dans les cas extrêmes, ils recourent pendant les sessions de rattrapage en général, c'est-à-dire l'étape de la dernière chance pour eux, à des candidats mercenaires qui quelques fois parviennent à déjouer tout l'arsenal de contrôle mis en place pour les substituer et composer pour eux.

Telles sont les principales conséquences qui découlent de la centration sur le français central sans insertion de la variation dans l'enseignement du FLE à l'université de Buéa. Insertion qui pourrait réduire considérablement l'hémorragie, faute de la stopper complètement.

\section{Les améliorations envisagées}

Les défaillances et les conséquences entraînées par la non intégration de la variation dans l'enseignement du FLE à l'université de Buéa incitent à dégager des améliorations pour résorber les pannes actuelles. Elles sont 
essentiellement d'ordre pédagogique et prennent en ligne de compte la variation linguistique d'une part et la variation culturelle d'autre part.

\section{Au niveau de la variation linguistique}

Avant d'aller plus loin, il convient de préciser que quelle que soit l'astuce proposée, il n'est en aucun cas question de prôner l'enseignement actif des différentes variétés de français du Cameroun et d'ailleurs. Il s'agit plutôt les intégrer en arrière plan des enseignements; d'en faire un second but de l'enseignement du FLE au Cameroun. A ce moment, l'objectif de l'enseignement du FLE ne se limite plus seulement à l'acquisition d'une quantité ou d'un volume de compétences communicatives tel que c'est d'actualité, mais bien plus à participer à l'éveil et à la prise de conscience linguistique des étudiants. Ce recentrage du but de l'enseignement des langues étrangères en faveur de la variation et que porte à cœur Valdman $(1975,2000)$ s'avère nécessaire pour le succès des activités d'enseignement/apprentissage. Son introduction subtile, mais effective dans l'enseignement du FLE à Buéa conduira les apprenants à prendre conscience des comportements langagiers, à comprendre et expérimenter les notions de tolérance linguistique et de tolérance tout court, prenant de ce fait le relais des langues maternelles.

Concrètement parlant, le but de ce type d'enseignement ne consiste pas à maîtriser les variantes du français du Cameroun ou d'autres pays francophones. Il s'agit plutôt pour l'enseignant de FLE de faire intervenir 306 implicitement les variations dans des documents déclencheurs authentiques écrits, visuels, sonores, audiovisuels sélectionnés judicieusement. L'observation, l'écoute, suivi d'autres manipulations modulées par l'enseignant permettront aux apprenants d'identifier et de comprendre les différences et d'être de la sorte plus disposés à cultiver des attitudes tolérantes et débarrassées de préjugés linguistiques à l'égard des locuteurs qui les emploieront. En réfléchissant et s'exerçant ainsi sur la variation linguistique dans un environnement plurilingue comme le Cameroun avec 283 d'après Bitja'a Kody (2004: 513-214), ils développent de façon générale la culture de l'objectivité vis-à-vis des langues et de leurs locuteurs, quels qu'ils soient.

Des expériences similaires, mais restreintes sont d'ailleurs entreprises en Norvège depuis quelques temps, au moyen de supports sonores et audiovisuels qui permettent aux apprenants d'acquérir quelques connaissances sur les variétés régionales, dialectales... L'unité de français fonctionnel du département de français de l'université de Buéa pourrait s'en inspirer afin d'opérer ses réaménagements avec plus d'efficacité

\section{Au niveau de la variation culturelle}

La langue n'étant qu'une composante de la culture, il n'y a qu'une enjambée franchie de l'une à l'autre. S'il faut faire la promotion de la variation linguistique en FLE, alors la variation culturelle devrait automatiquement lui emboîter le pas. Chaque communauté ou groupe linguistique possède une culture qui lui est propre, c'est-à-dire un ensemble de données, de principes, 
de conventions qui guident ou orientent ses comportements et sur lesquels, il s'appuie pour interpréter les comportements d'autrui. De là apparaît la notion d'interculturalité qui, de l'avis de (De Carlo, 1998: 40), «serait une solution aux contacts/conflits des langues-cultures imposés par une société multiculturelle puisqu'il induirait négociation et compromis ou une sorte d'inter-solidarité ». En tant qu'un des pays les plus linguistiquement et culturellement hétérogènes d'Afrique, le Cameroun s'insère justement dans cette logique. L'enseignement du FLE devient donc une occasion supplémentaire pour promouvoir la paix, l'unité et la tolérance à travers des transpositions didactiques de la notion d'interculturalité. Elle se traduit par des approches culturelles centrées non plus seulement sur la socioculture française cible tel que c'est le cas avec les manuels actuels, mais également sur celles des apprenants qui sont d'abord camerounais, mais aussi nigérians, ghanéens, équato-guinéens, centrafricains, tchadiens... pour les plus représentatifs. Cette démarche les rend plus attentifs, les stimule et les rend capables d'identifier les traits communs partagés par les langues et cultures de départ d'une part et celle d'arrivée d'autre part. Elle les dote aussi d'outils métacommunicatifs qui leur permettront d'être attentifs aux aspects interculturels dans leurs interactions dans des situations réelles, de réguler leurs échanges exolingues, de poursuivre leur apprentissage sur le terrain. $\mathrm{Ce}$ qui n'aurait pas été le cas s'ils s'étaient cantonnés uniquement aux manipulations relatives à la culture cible française. De même, ils prendront ensemble conscience des stéréotypes qui sont véhiculés dans les différentes cultures étudiées, les démystifieront et expérimenteront au finish le métissage culturel qui provient des productions collectives en intégrant des éléments propres à chaque culture et en se servant d'éléments culturels étrangers à chacun.

Avec la pédagogie de l'interculturalité, l'accent est également mis sur les apprenants qui sont pris pour qui ils sont, et non des êtres de fiction à qui on fait jouer des rôles. A partir par exemple des rituels communicatifs en contexte camerounais : la proxémique entre les petits et les plus âgés, les sujets et les chefs, entre enfants ou amis ; la kinésique, c'est-à-dire les gestes et les mimiques (le revers d'une main que l'on bat dans la paume de l'autre main pour supplier quelqu'un, la poitrine que l'on tape en signe d'orgueil, la tête que l'on tient des deux mains en signe de consternation, de désolation ou de malheur...), ils acquièrent les rudiments et les distances requises pour apprécier objectivement non seulement la culture française, mais toutes les autres cultures. Et c'est seulement à cet instant que le propos contenu dans la loi d'orientation de l'éducation au Cameroun qui voudrait former des Camerounais solidement encrés dans leurs cultures et aussi préparés pour s'ouvrir aux souffles intérieurs revêt tout son sens.

En outre, enseigner le FLE tel qu'il se fait à Buéa fournit un prétexte en béton aux Anglophones pour continuer de nourrir leur susceptibilité et pour continuer de marteler sur tous les toits qu'ils subissent une seconde colonisation. Lorsque les savoirs savants seront négociés en savoirs enseignables dans les nouveaux manuels de FLE, avec entre autres des noms originaires de leurs aires géographiques tels : Agbor, Mafani, Egbe, Nalova, Enongene, Titanji, Ebenye, Ojong, Ambe, Ndifor, Egbe, Limbe, Bakinguili, 
kumba, Bakassi, Mamfée, Buéa, Santa, Essungue, Molyko, Bessem, Bambili... ou encore des illustrations et des textes d'appui inhérents à leurs réalités socioculturelles bakossi, bafut, méta, bakwere, banyangue, bali, ..., ils seront plus sujets à accorder du crédit aux cours de FRE 101 et FRE 102. Ils baisseront alors la garde et pourront pleinement s'approprier les slogans « unity in diversity » ou encore «One and indivisible ! " que le Cameroun fait prévaloir. Étendue aux réalités d'autres pays, cette expérience les édifierait davantage, au même titre que les autres étudiants francophones et étrangers.

L'essentiel de ces suggestions tourne autour de la redéfinition de l'objectif assigné au FLE au profit de sa réorientation vers une approche variationniste. $\mathrm{Ce}$ qui rendrait les leçons plus mobilisatrices et permettrait de décongestionner les effectifs pléthoriques occasionnés par les échecs massifs des étudiants. Ce revirement limitera aussi les innombrables cas de tricherie et de mercenariat qui font le quotidien de l'université de Buéa. Mais pour que la mayonnaise prenne, il faudrait que la durée et le temps d'intervention didactique soient revus à la hausse, afin de déployer dans toute sa splendeur les pédagogies de variation linguistique et culturelle. En outre, la révision des manuels dans la perspective de leur adaptation à de nouveaux objectifs constitue également un impératif pour la réussite de cette entreprise. Il faudrait aussi homogénéiser autant que faire se peut les profils des apprenants; ce à travers des tests de présélection préalable et une identification des besoins réels des apprenants, car pour certains étudiants 308 issus du sous-système francophone dont le niveau est assez élevé en français, ces cours constituent une réelle perte de temps.

En addition, il faudrait aussi reconnaître à la suite de Salengros (2006) qu'internet s'impose comme étant un puissant support pédagogique dans la mise en évidence de la variation linguistique et surtout culturelle, de par l'abondante variété thématique, illustrative... offerte par les sites. Cependant, il serait plus prudent de ravaler son enthousiasme face à cet outil dans un contexte relativement pauvre comme celui du Cameroun, car son utilisation implique des contraintes pas toujours à la portée de tous les étudiants (mobiles, ordinaturs, électricité, connexion, etc.)

\section{Conclusion}

On retient de cette étude qu'à l'inverse de l'enseignement de l'anglais langue étrangère, l'enseignement du FLE à l'université de Buéa n'accorde pas de place à la variation linguistique et culturelle. Il a été donné de constater avec les programmes transposés dans les manuels que l'apprentissage était exclusivement orienté vers le contexte français et occidental. Il est à concéder avec Onguene Essono, (2003: 60) que la mission ultime de l'enseignant de français consiste à «faire maintenir la correction linguistique ». Pourtant, le choix du français central ou neutre comme norme à enseigner n'implique toutefois pas le rejet total de la variation dans l'enseignement du FLE. En effet, il n'est pas possible pour le français normé de remplacer ses usages qui en fait, ne sont que des emplois contextuels qui constituent la norme endogène du fait de leur popularité ou de leur longévité. Et parce que ces 
usages traduisent avec plus d'exactitude les réalités socio-culturelles que vivent les Camerounais dans leurs différentes régions, ils assument aussi des fonctions identitaires qui méritent d'être prises en considération. Un avis que partage que Dassi (200: 114), c'est pourquoi il met un point d'honneur à éclaircir que «Le français de référence c'est celui du commun des Francophones. C'est donc le français qui fonde la francophonie linguistique [...] La norme ne saurait se confondre avec un usage, fût-elle homologuée, en même temps qu'elle ne saurait s'y opposer ", comme pour dire qu'une relation de partenariat à bénéfices mutuels est indispensable entre la norme et les usages du français pour la survie et l'épanouissement du français en francophonie. Une telle collaboration garantit un enseignement approfondi de la langue.

Au micro niveau de l'université de Buéa, la variation linguistique permettra aux étudiants que l'on y retrouve d'être plus conscients de leurs comportements langagiers à travers leur contextualisation dans leurs environnements immédiats et cibles. Ce qui les dépouillera des préjugés et les cultivera à l'objectivité et à tolérance. À la strate culturelle, la variation modèlera des étudiants Camerounais francophones, anglophones et étrangers de l'université de Buéa à la citoyenneté mondiale et au patriotisme en même temps, tel que le conçoit Dumont (2008: 8) lorsqu'il déclare: " L'interculturel, c'est faire face à l'Autre, non pas pour l'affronter mais pour le compléter, pour vivre en parallèle avec lui, l'écouter, s'ouvrir, construire le dialogue avec lui. Toutes les cultures sont égales, s'observent, s'inspirent mutuellement. L'interculturel, ce sont des langues-cultures qui se croisent et qui veulent se comprendre ».

Il est donc question de revisiter les manuels en usage dans l'optique d'un arrimage aux nouvelles donnes. Mais pour que toutes les conditions de félicité soient réunies, il importe sérieusement d'homogénéiser les groupes, d'accroître l'enveloppe horaire des cours, revoir la taille des groupes trop touffus pour une bonne lisibilité et la prise en main efficaces de l'enseignement, mettre à la disposition des apprenants dont le niveau de vie demeure moyen, des facilités mises à jour (laboratoires de langues, salle multimédia, médiathèques...)

\section{Bibliographic references}

BEACCO, J.-C. 2000. Les dimensions culturelles des enseignements de langue. Paris. Hachette.

BIRAGO DIOP. 1960. Souffle des ancetres. In Leurres et Lueurs. Presence africaine.

BITJA'A, KODY, D. Z. 2004. La Dynamique des langues camerounaises en contact avec le français: Approche macrosociolinguitistique. Thèse de doctorat $3^{\mathrm{eme}}$ cycle. Université de Yaounde I.

CALVET, L. J. 2002. Sociolinguistique. Paris. Nathan. $4^{\text {eme }}$ edition revue et corrigee.

DASSI, M. 2009. Norme du français de reference et abstraction. In Revue internationale des arts lettres et sciences sociales (RIALSS), vol 3. Yaoundé. Africana Publications, pp. 114-123.

De CARLO, M. 1998. L'Interculturel. Paris.Cle-international, 128 p. 
DEWAELE, J-M. - MOUGEON R. 2002. L'acquisition de la variation par les apprenants du français langue seconde. AILE, n. 17.

ELOY, J. M. 2003. Enseigner le français avec la variation. In Travaux de didactique de FLE. Publications Montpellier 3. IEFE. Universite Paul Valery, n. 49. pp.1-14.

FAVART, M. 2010. Quels savoirs en matiere de variations langagieres susceptible d'optimiser un enseignement du FLE ? In Pratiques, n. 145/146, pp. 179-196.

GADET, F. 2003. La variation sociale en français. Paris: Ophrys.

LABOV, W. 1976. La Sociolinguistique. Paris. Minuit. Traduit de l'anglais par Alain Kihm. Collection Le Sens Commun.

LODGE, A. 1993. French: from dialect to standard. London: Routledge.

MBONDJI-MOUELLE, M. M. 2012. Plurilinguisme et partenariat linguistique en didactique du français langue seconde (FLS) au Cameroun. In Syllabus Review. 3 (1), pp. 127-152.

MODO ASSE. 2008. Un Exemple de communication commerciale en Afrique. La relation «asso» sur la place des marches camerounais. In Revue internationale des arts lettres et sciences sociales (RIALSS), vol 3. Yaounde. Africana Publications, pp. 133-150.

NGALASSO, M. M. 1990. Pour une didactique comparative du français et de langues africaines. In Diagonales et cultures, n. 34, pp. 15-26.

ONGUENE ESSONO, L. M. 2003. Normes en eclats pour un français correct au Cameroun. In Langues et communication. Revue scientifique de recherche multidisciplinaire, vol. 1, n. 3. U.Y.I. 2 (3), pp. 57-72.

PIEBOP, G. M. C. 2015. Reticences des camerounais de culture anglophone a l'apprentissage du français. In l'Enseignement du français en zone anglophone au Cameroun. Kansas City. Miraclaire Academic publication, in association with Ken scholars publishings Raytown, pp. 143-164.

SALENGROS, I. 2006. Approche culturelle et Internet en classe de FLE : exemple d'une pratique a l'ecole nationale des ponts et chaussees (ENPC). In Alsic, vol. 9, pp. 161-180.

VALDMAN, A. 1975. Variation linguistique et norme pedagogique dans l'enseignement du français langue seconde. In Le Bulletin de la federation internationale des professeurs de français, 12-13: 667-706.

VALDMAN, A. 2000. Comment gerer la variation dans l'enseignement du français langue etrangere aux Etats-Unis. In The French Review, vol.75, n.4. VERDELHAN-BOURGADE, M. 2002. Le français langue de scolarisation. Pour une didactique realiste. Paris. PUF.

\author{
C. M. Gisele Piebop \\ University of Yaounde I \\ Faculty of Arts, Letters and Social Sciences \\ Department of French, Yaounde \\ Cameroon \\ giselepiebop@live.fr \\ gpiebop@yahoo.fr
}

